\title{
SOLVING FOR MOTION AND ACTIVATION SIMULTANEOUSLY IN AN FMRI EXPERIMENT WITH MULTIPLE STIMULUS CONDITIONS
}

\author{
J. Orchard $^{*}$ \\ University of Waterloo \\ School of Computer Science \\ Waterloo, ON, N2L 3G1 Canada
}

\author{
M. S. Atkins ${ }^{\dagger}$ \\ Simon Fraser University \\ School of Computing Science \\ Burnaby, BC, V5A 1S6 Canada
}

\begin{abstract}
It has recently been shown that greater accuracy in fMRI processing can be achieved if registration and activation detection are solved simultaneously rather than in sequence. However, this simultaneous solution has been demonstrated only for fMRI experiments in which a single stimulus condition was used. This paper presents results obtained by applying the simultaneous solution technique to simulated datasets with two stimulus conditions, and demonstrates that the technique is equally effective on such datasets.
\end{abstract}

\section{INTRODUCTION}

In fMRI, the processing steps of registration and activation detection are coupled. It is well established that patient motion must be removed from the dataset before activation detection can be accurately achieved $[1,2]$. On the other hand, it has recently come to light that activation should be removed from the dataset in order to achieve accurate registration. Freire et al. [3] hypothesized that the active voxels were behaving like outliers in the least-squares registration process, resulting in a systematic activation bias in the motion estimates. These motion errors ultimately lead to false-positive and false-negative activations in the resulting activation maps.

The fact that the two problems are coupled means they cannot accurately be solved independently. A method to solve the two problems simultaneously has been proposed [4], and uses a single data model designed to account for the effects of both motion and activation. The model combines a linearization of the rigid-body motion transformation, and the general linear model for activation. By representing an entire dataset in one matrix, the model can be expressed as $\mathbf{F} \approx \mathbf{G}+\mathbf{A X}+\mathbf{Y B}$, where $\mathbf{F}$ is a matrix that holds the entire observed dataset with volumes stored in columns, and

\footnotetext{
* The work of J. Orchard was funded in part by the Natural Science and Engineering Research Council of Canada.

${ }^{\dagger}$ The work of M.S. Atkins was funded in part by the Natural Science and Engineering Research Council of Canada.
}

voxel time series stored in rows. The matrix $\mathbf{G}$ is a motionless and activationless baseline dataset. The term $\mathbf{A X}$ is the linear motion term in which the matrix $\mathbf{X}$ holds the time series of the 6 motion parameters, one in each row, and A holds the appropriate derivatives of the baseline volume with respect to the motion parameters in its columns. The term YB is the activation term, and is also split into a spatial component ( $\mathbf{Y}$ which holds $s$ activation maps, one in each column), and a temporal component (B which holds $s$ stimulus regressors, one in each row). Stated as a least squares problem, we have

$$
\min _{(\mathbf{X}, \mathbf{Y})}\|\mathbf{A X}+\mathbf{Y B}-\mathbf{C}\|
$$

where $\mathbf{C}$ is simply $(\mathbf{F}-\mathbf{G})$, and $\|\cdot\|$ is the Frobenius norm.

Methods to solve (1) for the motion (X) and activation (Y) simultaneously were proposed in [4] and [5]. However, neither of these publications present results pertaining to fMRI experiments with more than one stimulus condition. Here, we show that the simultaneous model, and the solution outlined in [4], is capable of overcoming the activation bias and achieve accurate activation maps with two stimulus conditions.

\section{THEORY}

The least-squares problem in (1) can be solved by taking the QR decomposition of the matrix $\mathbf{B}^{\prime}$ (the transpose of $\mathbf{B}$ ) [4]. Let $\mathbf{Q}_{1}, \mathbf{Q}_{2}$ and $\mathbf{R}$ be defined according to the equation

$$
\mathbf{B}^{\prime}=\left[\begin{array}{ll}
\mathbf{Q}_{1} & \mathbf{Q}_{2}
\end{array}\right]\left[\begin{array}{c}
\mathbf{R} \\
0
\end{array}\right]
$$

where $\mathbf{R}$ is $s \times s$ and upper-triangular. The simultaneous solution to (1) can be stated analytically as

$$
\begin{aligned}
& \mathbf{X}=\mathbf{A}^{\dagger} \mathbf{C Q}_{2} \mathbf{Q}_{2}^{\prime} \\
& \mathbf{Y}=\mathbf{C Q}_{1}\left(\mathbf{R}^{\prime}\right)^{-1}
\end{aligned}
$$

where $\mathbf{A}^{\dagger}$ is $\left(\mathbf{A}^{\prime} \mathbf{A}\right)^{-1} \mathbf{A}^{\prime}$, the matrix pseudo-inverse of $\mathbf{A}$. 
It should be noted that nowhere in the derivation of the solution (equations (3) and (4)) is there any constraint on $\mathbf{B}$ other than that $\mathbf{B}$ have full rank. If $\mathbf{B}$ does not have full rank, this means that the fMRI experiment is poorly designed, and the breakdown in the solution is due to a mathematical flaw in design, not the algorithm for finding the solution. If $\mathbf{B}$ has full rank, the resulting $\mathbf{R}$ is $s \times s$ (when there are $s$ stimulus regressors), and equation (4) produces estimates for $s$ activation maps, as required. Thus, the same solution applies to datasets with multiple stimulus conditions, even though it has not previously been demonstrated.

The problem stated in (1) does not have a unique solution. Consider the following derivation:

$$
\begin{aligned}
& \min _{(\mathbf{X}, \mathbf{Y})}\|\mathbf{A X}+\mathbf{Y B}-\mathbf{C}\| \\
= & \min _{(\mathbf{X}, \mathbf{Y})}\|\mathbf{A} \mathbf{X}+\mathbf{Y B}-\mathbf{C}+\mathbf{A} \alpha \mathbf{B}-\mathbf{A} \alpha \mathbf{B}\| \\
= & \min _{(\mathbf{X}, \mathbf{Y})}\|\mathbf{A}(\mathbf{X}+\alpha \mathbf{B})+(\mathbf{Y}-\mathbf{A} \alpha) \mathbf{B}-\mathbf{C}\| .
\end{aligned}
$$

Thus, if $(\mathbf{X}, \mathbf{Y})$ is a solution, then so is $(\mathbf{X}+\alpha \mathbf{B}, \mathbf{Y}-\mathbf{A} \alpha)$ for any $6 \times s$ matrix $\alpha$. However, as long as we can find any solution at all, we can simply adjust $\alpha$ to our liking. In [4], an additional constraint,

$$
\min _{\alpha} \sum_{r} \arctan \left(k\left|[\mathbf{Y}-\mathbf{A} \alpha]_{r}\right|\right),
$$

was used to remove unwanted motion artifacts from the activation maps and arrive at a satisfactory solution to the simultaneous problem. The subscript $r$ in (8) denotes the $r$ th voxel.

\section{METHODS}

Both the standard least-squares method and the simultaneous method were implemented in C++ using Fourier resampling [6,7]. An altered form of the constraint in (8) was used to address the fact that there are now two activation maps, one for each stimulus.

$$
\min _{\alpha} \sum_{r} \arctan \left(k\left|[\mathbf{Y}-\mathbf{A} \alpha]_{r, c}\right|\right), c=1,2
$$

The subscripts $r$ and $c$ denote row and column, respectively. A value of 0.05 was used for $k$. This parameter determines the sensitivity (or lack thereof) to outliers. It is important to note that while (9) is a minimization over all the columns of $\alpha$, each column can actually be solved for independently. Thus, the processing time increases linearly with respect to the number of stimulus conditions.

The simultaneous method was compared to the standard least-squares method (in which registration is done first, followed by activation detection). To assess the robustness of the two methods, they were each used to analyze a set of
40 different simulated fMRI datasets. These datasets were generated using 80 copies of a single EPI volume with dimensions $64 \times 64 \times 30$. The voxel dimensions were $3.75 \times$ $3.75 \times 4(\mathrm{~mm})$. Two different stimulus functions, stimulus 1 and stimulus 2 , were created from slightly smoothed boxcar functions. Stimulus 1 was non-zero for frames 6-16, 26$36,46-56$, and 66-76. Stimulus 2 was non-zero for frames 11-32 and 51-72. For each stimulus, an activation map was created manually. The region of activation for stimulus 1 occupies a large region in the occipital and temporal lobes, while the region of activation for stimulus 2 occupies a large region in the frontal lobes. After applying the activation and motion, the datasets were corrupted with additive Gaussian noise (standard deviation of $2.5 \%$ of the mean brain intensity), and were blurred with a 5-mm (full-width at half-max) Gaussian kernel.

The datasets were split up into four different types as follows:

- 10 datasets included activation and random motion,

- 10 datasets included activation and stimulus-correlated motion,

- 10 datasets included stimulus-correlated motion and no activation, and

- 10 datasets included activation but no motion.

The stimulus-correlated motion profiles were generated using a random weighting of a random walk, and the two stimulus functions.

Both the standard least-squares method and the simultaneous method were used to estimate the motion for all 40 datasets. These motion estimates were then used to resample the datasets (using Fourier interpolation $[6,7]$ ) before least-squares activation detection. In the activation detection, both a linear fit coefficient and a correlation coefficient were calculated. These activation maps were thresholded so that only voxels with a fit coefficient greater (in absolute value) than 40 , and a correlation coefficient greater (in absolute value) than 0.363 were considered to be active and included in the final activation mask. The resulting binary activation masks were used to calculate false-positive and false-negative activation rates. The gold standard activation mask was established by performing the same activation detection process (but not registration) on a simulated dataset that contained no motion corruption.

\section{RESULTS}

Figure 1 shows how the errors in the motion estimates differ between the standard least-squares registration method and the simultaneous solution. The motion plots show the error 
htb
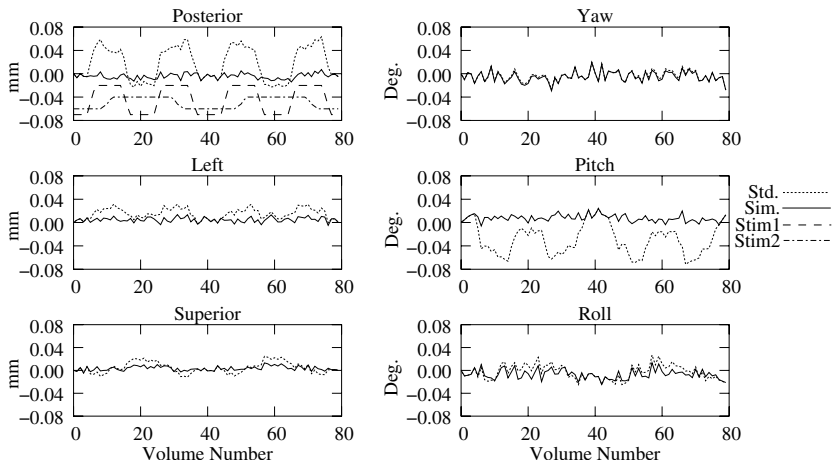

Fig. 1. Errors in the motion parameter estimates for a motion-corrupted dataset with activaiton. The standard least-squares method ("Std.") exhibits errors that are correlated to the stimulus function, while the simultaneous method ("Sim.") does not. The two stimulus functions are shown in the posterior translation plot.

in the estimates for a simulated dataset that contains activation and random motion. The standard method error plots show a high degree of similarity to the stimulus functions. Moreover, many of the standard method plots appear to be a linear combination of the stimulus functions (plus noise). For comparison, the two stimulus functions are also plotted in the posterior translation graph.

The activation maps corresponding the dataset used in Fig. 1 are shown in Fig. 2 and Fig. 3. Note the regions of false activation near the edges of the brain in Fig. 2 (b). These are false-positive activations.

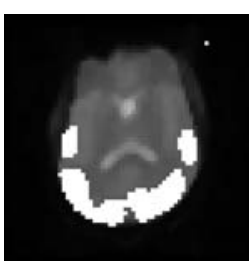

(a) True

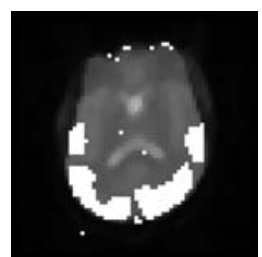

(b) Std.

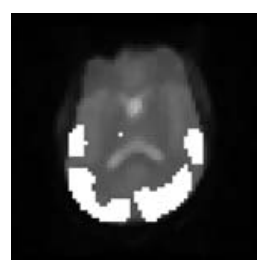

(c) Sim.
Fig. 2. Activation masks corresponding to stimulus 1 for a simulated dataset that contains activation and random motion. The standard least-squares method results in regions of false activation (b), whereas the mask produced by the simultaneous method (c) looks much more like the true activation mask (a).

Table 1 lists the false-positive and false-negative activation rates for the two analysis methods, averaged over the 10 simulated datasets for each of the 4 dataset types. For stim-

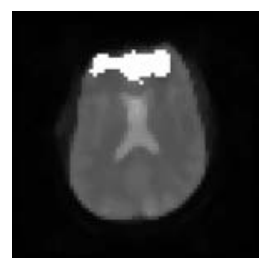

(a) True

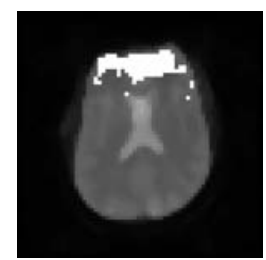

(b) Std.

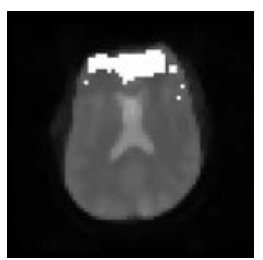

(c) Sim.
Fig. 3. Activation masks corresponding to stimulus 2 for a simulated dataset that contains activation and random motion. Although the activation masks look similar, they are not quite the same. Table 1 reports the differences in false activation rates.

ulus 1, the simulated method shows far fewer false-positive and false-negative activations than the standard method. For datasets with activation, the standard method averaged about $50 \%$ more false-positives and about $45 \%$ more false-negatives than the simultaneous method. The results for stimulus 2 are not as extreme. In fact, the standard method shows about $10 \%$ fewer false-positives, but $30 \%$ more false-negatives, than the simultaneous method.

\section{DISCUSSION AND CONCLUSIONS}

The simultaneous solution method is capable of distinguishing motion artifact components from activation components in simulated fMRI datasets. This was previously shown for fMRI experiments with only one stimulus condition. Here, we have demonstrated that it works equally well for 2 stimulus conditions. We are confident that it will also work for even more stimuli.

A comparison of activation masks produced by the two methods shows that the standard least-squares method results in substantially more false activations for stimulus 1 , and moderately more for stimulus 2 .

The simultaneous method requires no extra coding over and above the method outlined in [4]. The only difference is that the constraint needs to be applied for each stimulus condition. These constraints can be applied independently and result in only a linear increase in computational time. The simultaneous method is modest in its use of memory and CPU resources. The technique takes only about twice as long as the standard method to solve for $\mathbf{X}$ and $\mathbf{Y}$ simultaneously.

The simultaneous model and solution method show great promise, and we are currently investigating its application to non-rigid registration, as well as its effectiveness on in vivo datasets. 
Table 1. False-positive and false-negative activation counts for the two analysis methods, averaged over 10 datasets for each dataset type. The true activation masks contain 3252 active voxels for stimulus 1, and 2233 active voxels for stimulus 2.

\begin{tabular}{|c|c|c|c|c|c|c|c|c|}
\hline \multirow[b]{3}{*}{ Dataset Type } & \multicolumn{4}{|c|}{ Stimulus 1} & \multicolumn{4}{|c|}{ Stimulus 2} \\
\hline & \multicolumn{2}{|c|}{ False-Positives } & \multicolumn{2}{|c|}{ False-Negatives } & \multicolumn{2}{|c|}{ False-Positives } & \multicolumn{2}{|c|}{ False-Negatives } \\
\hline & Std. & Sim. & Std. & Sim. & Std. & Sim. & Std. & Sim. \\
\hline activation, random motion & 291.5 & 196.5 & 289.2 & 202.7 & 109.7 & 121.6 & 161.7 & 124.5 \\
\hline activation, stim-corr motion & 296.3 & 201.4 & 286.4 & 200.8 & 113.3 & 121.5 & 161.0 & 124.8 \\
\hline no activation, stim-corr motion & 16.8 & 17.1 & 0.0 & 0.0 & 12.0 & 12.2 & 0.0 & 0.0 \\
\hline activation, no motion & 283.2 & 178.0 & 287.5 & 189.8 & 92.1 & 104.8 & 156.1 & 114.7 \\
\hline
\end{tabular}

\section{REFERENCES}

[1] J. V. Hajnal, R. Myers, A. Oatridge, J. E. Schwieso, I. R. Young, and G. M. Bydder, "Artifacts due to stimulus correlated motion in functional imaging of the brain," Magn Reson Med, vol. 31, pp. 283-291, 1994.

[2] N. F. Ramsey, J. S. van den Brink, M. M. C. van Muiswindle, P. J. M. Folkers, and C. T. W. Moonen, "Phase navigator correction in 3D fMRI improves detection of brain activation: Qualitative assessment with a graded motor activation procedure," NeuroImage, vol. 8, pp. 240-248, 1998.

[3] L. Freire and J.-F. Mangin, "Motion correction algorithms of the brain mapping community create spurious functional activations," in Proceedings of Info Proc Med Imaging, Davis, June 2001, pp. 246-258.

[4] J. Orchard, C. Greif, G. Golub, B. Bjornson, and M. S. Atkins, "Simultaneous registration and activation detection for fMRI," IEEE Trans. Med. Imag., 2003, (in press).

[5] J. Orchard and M. S. Atkins, "Iterating registration and activation detection to overcome activation bias in fMRI motion estimates," Medical Image Computing and Computer Assisted Intervention (MICCAI), 2003, (in press).

[6] R. W. Cox and A. Jesmanowicz, "Real-time 3D image registration for functional MRI," Magn Reson Med, vol. 42, pp. 1014-1018, 1999.

[7] W. F. Eddy, M. Fitzgerald, and D. C. Noll, "Improved image registration using fourier interpolation," Magn Reson Med, vol. 36, no. 6, pp. 923-931, 1996. 